\title{
A qualitative study exploring the role of community health workers in promoting maternal postpartum mental health in Nicaragua
}

\author{
Anna Steeves-Reece ${ }^{1}$, Nicole Yuan², Linda Green ${ }^{3}$ \\ 1 Oregon Health \& Science University-Portland State University School of Public Health, Portland, Oregon, USA, 2 University of Arizona, Mel \& Enid \\ Zuckerman School of Public Health, Tucson, Arizona, USA, ${ }^{3}$ University of Arizona, School of Anthropology, Tucson, Arizona, USA \\ Keywords: maternal health, nicaragua, global health
}

https://doi.org/10.29392/joghr.3.e2019089

Journal of Global Health Reports

Vol. 3, 2019

\section{Background}

The objectives of this qualitative study were to examine perceptions of maternal postpartum distress among community health workers and mothers living in rural Nicaragua; and to identify the role of community health workers (CHWs) in implementing locally relevant strategies to reduce maternal postpartum distress.

\section{Methods}

The study was conducted between May and July 2015 with a small, rural community in the central highlands of Nicaragua. Primary data sources included two focus group interviews with 10 community health workers, and individual interviews with 12 mothers who had delivered their most recent child within roughly the previous year.

\section{Results}

Both community health workers and mothers identified maternal distress following childbirth as a community concern. Identified factors that contributed to postpartum distress were lack of people to confide in, experiences of intimate partner violence, and exposure to household poverty. Participants felt that community health workers might play an important role in ameliorating postpartum distress by providing social support to mothers and engaging intimate partners to offer assistance. However, results indicated that any community health worker interventions must also prioritize the well-being of CHWs.

\section{Conclusions}

Community health workers have the potential to address gaps in postpartum services for mothers living in rural Nicaraguan communities. However, such programs must consider the well-being of CHWs, and should be developed along with interventions and policies that address major social factors, such as poverty and interpersonal violence, to promote the mental health of mothers in rural Nicaragua.

Postpartum mental health must be considered when promoting the well-being of mothers and children around the globe. It is well documented that some women experience distress following childbirth in the forms of postpartum depression, anxiety, or stress. ${ }^{1,2}$ In addition to a mother's suffering, postpartum distress may contribute to negative consequences for children. Postpartum depression is associated with impaired bonding between mothers and infants, which can lead to physical, emotional, and social problems. ${ }^{3-6}$

Postpartum distress, including postpartum depression, is particularly concerning for low- and lower-middle-income countries (LMICs). A systematic review of perinatal mood disorders in LMICs found the prevalence of mood disorders after childbirth was $19.8 \%$ for the countries reviewed. ${ }^{7} \mathrm{~A}$ systematic review of high-income countries documented lower prevalence rates, with major and minor depression af- ter childbirth ranging from $6.5 \%$ to $12.9 \% .{ }^{8}$ Postpartum distress in LMICs is attributed to multiple factors, including poverty, intimate partner violence, inadequate practical or emotional support, and adverse life events. ${ }^{7}$

Challenges of postpartum distress in LMICs may be exacerbated by few or inaccessible mental health services. ${ }^{9}$ To address this problem, some mental health interventions have integrated community health workers (CHWs). ${ }^{10}$ CHWs are often described as trusted community members who serve as liaisons between formal health services and communities. ${ }^{11}$ Various global health systems have incorporated CHWs to prevent and/or mitigate maternal mental illnesses, including those in South Africa, Jamaica, Zimbabwe, and Pakistan. ${ }^{12-15}$ Latin American countries have used CHWs for decades, ${ }^{16}$ but little is known about how CHWs may promote maternal mental health in the region. 


\section{RATIONALE FOR RESEARCH IN NICARAGUA}

Perinatal distress is a public health concern in Nicaragua. One study with pregnant women in the south of Nicaragua found $41 \%$ had symptoms of anxiety and $57 \%$ had symptoms of depression. ${ }^{17}$ A study with the participating rural community of the current study found $24 \%$ of mothers had levels of symptom severity indicative of common mental disorders. ${ }^{18}$ Similar to other LMICs, Nicaraguan mothers are exposed to social and economic hardships that may increase their vulnerability to postpartum distress, including food insecurity, ${ }^{18,19}$ which is a correlate of poverty, and intimate partner violence. ${ }^{20-22}$

Despite potentially high occurrences of postpartum distress, there is a dearth of mental health resources in Nicaragua. One study found less than $25 \%$ of Nicaraguans had access to any mental health services. ${ }^{23}$ While $9 \%$ of the country's GDP is dedicated to health, ${ }^{24}$ only $1 \%$ of that is for mental health. ${ }^{25}$ An asset of Nicaragua's health system is its collaboration with CHWs which are referred to as brigadistas. Brigadistas, most of whom are unpaid volunteer workers, have played a vital role in strengthening health care for urban and rural populations since the early 1980s. ${ }^{26}$ One study found that they volunteer roughly 75 hours per month. Their activities focus on maternal health, including encouraging pregnant women to travel to clinics for prenatal checks and to deliver their babies. ${ }^{27,28}$ While the Nicaraguan Ministry of Health (MINSA) standardizes most work completed by CHWs, their roles and responsibilities vary depending on community needs and the presence of additional organizations, like NGOs, who sometimes collaborate with them. To inform the role of brigadistas' in maternal mental health, the current study had two goals. The first was to examine perceptions, including the perceived causes, of postpartum distress among mothers and CHWs in a rural community in Nicaragua. The second goal was to identify CHWs' role in implementing locally relevant strategies to reduce postpartum distress.

\section{MATERIALS AND METHODS}

\section{STUDY SITE}

The study was conducted with a rural community in the department (i.e., province) of Jinotega. The department is known for its coffee production and has a population of 445,000 . Most people live in rural settings. ${ }^{29}$ The community was selected because it has a strong network of CHWs that partner with both MINSA and a local non-governmental organization (NGO). The lead researcher had lived in Nicaragua for three years (2011 to 2014) as a maternal and child health promoter in collaboration with MINSA. During that time, she also worked with the local NGO and CHWs on a water filtration project.

\section{DATA COLLECTION}

Two data sources were collected from May to July 2015, including focus group interviews with $\mathrm{CHWs}$ and individual interviews with mothers who had delivered their most recent child within roughly the previous year. The lead re- searcher conducted all focus group and individual interviews. Interview guides were constructed with input from the research team consisting of three individuals with expertise in public health, anthropology, qualitative methods, and Latin American studies. Interview guides were revised based on feedback from Nicaraguan informants, including a MINSA nurse, a Nicaraguan psychologist working for a local NGO, and a CHW in the community. The individuals provided feedback to ensure the cultural relevance of the questions. It became apparent the term "postpartum depression" (depresión posparto) would be inappropriate and ineffective in the local context because depression or sadness associated with childbirth had not been widely medicalized in rural Nicaragua. That is, while community members may be concerned about postpartum distress, they may not view that sadness in terms of a specified disease. Therefore, interview guides for CHWs and mothers used the expression "tristeza después del parto" or "sadness following childbirth."

Focus group interviews were conducted with CHWs who worked simultaneously with MINSA and the local NGO. Based on her experiences working with pregnant women in Nicaragua and a literature review, the lead researcher consulted with NGO leadership and one of the CHWs to describe the proposed study and assess whether the topic was relevant to community needs. The individuals thought the topic was pertinent to the community and invited the lead researcher to present the project at a CHW monthly meeting and invite CHWs to participate. The NGO organized monthly meetings with the CHWs to review and coordinate health promotion activities. During the presentation, the lead researcher emphasized participation was optional, and that CHWs could opt out at any time for any reason. She used a sign-up sheet for recruitment, and followed up with individuals by telephone.

Twelve CHWs signed up at the meeting, and 10 ultimately participated in two focus group interviews (see Table 1). Two of the CHWs who originally signed up could not attend due to scheduling conflicts. One group had four individuals, and the other had six individuals. As presented in Table 1, nine out of 10 CHWs were female. The average age was 34 years old. The average length of time they had worked as a CHW was roughly 8 years, and the range was less than a year to 27 years. Both focus group interviews were conducted in a CHW's home and were facilitated by the lead researcher in Spanish. The interviews lasted about 90 minutes. The interview questions addressed perceptions of causes and protective factors for sadness after childbirth and strategies for CHWs to provide support to mothers.

Following the completion of the focus group interviews, individual interviews were conducted with mothers who lived in the community and had given birth during roughly the previous year. CHWs volunteered to recruit mothers who lived in their neighborhoods. The lead researcher trained the CHWs to provide brief descriptions of the study and interview process to mothers. Each CHW visited one to two mothers in their homes, described the study, and asked if they would be willing to participate. Twelve mothers told the CHWs they would participate in interviews. The CHWs then identified what day would work best for each mother and introduced them to the lead researcher. 
Table 1. Demographics of Community Health Workers

\begin{tabular}{lcccc}
\hline Participant & Gender & Age & Years of Education & Years as CHW \\
\hline 1 & F & 34 & 11 & 10 \\
\hline 2 & F & 31 & 4 & .25 \\
\hline 3 & F & 47 & 6 & 3 \\
\hline 4 & F & 26 & 2 & 4 \\
\hline 5 & F & 40 & 8 & 27 \\
\hline 6 & F & 36 & 6 & 8 \\
\hline 7 & F & 66 & 3 & 20 \\
\hline 8 & F & 33 & $11+$ & 5 \\
\hline 9 & F & 16 & 10 & 1.5 \\
\hline 10 & M & 16 & 9 & .25 \\
\hline
\end{tabular}

Table 2. Demographics of mothers

\begin{tabular}{lccccc}
\hline Participant & Age & Years education & Children & Age of youngest child & Where youngest child was born \\
\hline 1 & 39 & 7 & 6 & 9 months & Hospital \\
\hline 2 & 26 & 11 & 2 & 6 months & Hospital \\
\hline 3 & 24 & 3 & 2 & 14 months & Hospital \\
\hline 4 & 28 & 8 & 3 & 5 months & Hospital \\
\hline 5 & 30 & 0 & 2 & 1 month & Hospital \\
\hline 6 & 23 & 7 & 2 & 2 months & Hospital \\
\hline 7 & 23 & $11+$ & 1 & 7 months & Hospital \\
\hline 8 & 31 & 6 & 4 & 10 months & Hospital \\
\hline 9 & 18 & 1 & 1 & 3 months & Home \\
\hline 10 & 18 & 6 & 1 & 3 months & Hospital \\
\hline 11 & 19 & 3 & 1 & 9 days & Home
\end{tabular}

All 12 mothers who initially agreed to participate completed interviews. As presented in Table 2, the average age of the mothers was 25 , with a range of 18 to 39 . The mothers had between one and six children, and their education level ranged from no schooling to a technical university degree. The individual interviews took place either in the mothers' homes or a private location in the health clinic. They were conducted in Spanish by the lead researcher and lasted about 30 minutes. The interview questions focused on thoughts about postpartum sadness, social support following childbirth, resources for local mothers, and the role of CHWs in supporting women after childbirth.

\section{DATA MANAGEMENT AND ANALYSIS}

All focus group and individual interviews were audio recorded and transcribed verbatim in Spanish. Transcripts were read repeatedly to inductively identify codes related to the two research questions: 1 ) perceived causes of maternal postpartum distress, and 2) ways to mitigate postpartum distress, including the role of CHWs. A codebook was created for use with both the focus group and individual interviews. Inter-rater reliability of the coding was conducted between the lead researcher and another member of the re- search team. Both individuals coded a transcript of one interview (that the lead researcher had translated into English) independently and compared assigned codes. Discrepancies were discussed until final codes were established. The lead researcher coded the remaining transcripts in Spanish using the software program NVivo. Codes were further analyzed by the lead researcher and grouped into six themes that corresponded with the two research questions. To increase the validity of the findings, the results were shared with the participating CHWs during a return visit to the community in March 2016. CHWs confirmed the findings reflected their perceptions of local beliefs and needs related to postpartum distress.

\section{STUDY ETHICS AND INFORMED CONSENT}

The University of Arizona's Institutional Review Board (IRB) reviewed the study and determined it was exempt from further IRB oversight because of low risks to participants. Verbal consent was obtained from each participant prior to conducting each focus group and individual interview. 


\section{RESULTS}

Findings from interviews with CHWs and mothers were combined because questions and emergent themes were similar. CHWs and mothers reported observing postpartum distress in the community. According to one CHW:

"We have visited [women] that say they feel sad after giving birth, and that the easiest thing would be to take their own life."

Although interview questions focused on observations of other women in the community,

some participants disclosed personal experiences of postpartum distress.

\section{COMMUNITY PERCEPTIONS OF POSTPARTUM DISTRESS}

For each of the major research questions, three themes emerged. For the question about community perceptions of postpartum distress, the themes included: a lack of people to confide in, intimate partner violence, and exposure to household poverty.

Theme 1: Lack of people to confide in. To maintain good mental health, participants from both groups said women must be able to "desahogarse" (unburden oneself from negative thoughts/emotions or blow off steam) by talking with someone. Participants identified barriers to having someone to confide in. First, some women's partners may be absent because they either abandoned the family or work in far away places. Many participants shared the perspective of a CHW who said:

"One of the causes [of sadness] is that the mother, when she's giving birth, is alone. Her partner isn't accompanying her."

It is noteworthy, however, that several CHWs and mothers indicated their own partners were supportive and offered compassion and assistance with household chores after childbirth.

A second barrier was lack of trust with some health professionals. A few mothers described experiencing emotional abuse from health center staff. One mother stated:

$$
\begin{aligned}
& \text { "[Women] have told me they feel sad. Well, that they } \\
& \text { leave the hospital because they say [health care } \\
& \text { providers] mistreat them, that during childbirth they're } \\
& \text { rude to them." }
\end{aligned}
$$

Negative interactions in clinical settings contributed to women's reluctance to seek support from health care professionals.

A third barrier to having someone to confide in was gossip. CHWs and mothers described negative impacts of gossip, including concerns about what other people think about them. One mother said she hardly left her home because she did not want people to assume she was unfaithful to her partner. Another mother said:

"You can't trust anyone ... there are lots of people who
are not discreet. Well actually, the majority of people
are indiscreet."
Theme 2: Intimate partner violence. CHWs and mothers said mistreatment by intimate partners, including physical violence, sexual violence, and emotional abuse, contributed to distress. A CHW described "ugly stories" she heard from mothers when she provided family planning in their homes:

"Their husbands mistreat them in the home ... They
would tell me their husbands obligated them to have
sex [shortly after childbirth] ... To not be abandoned,
out of fear, they would comply."

Some CHWs reported personal experiences of intimate partner violence. One $\mathrm{CHW}$ disclosed her previous partner drank alcohol and beat her. The violence escalated to the point she had a miscarriage. She said leaving that partner made her life more tranquil, but she continued to experience negative memories from that period of time.

Mothers also shared personal stories of victimization. One mother said:

"The father [of my child] does not come here because if he does they will arrest him for the bad things he's done to me ... He threw me on the ground and kicked me while I was pregnant."

Theme 3: Exposure to household poverty. Participants from both groups reported that exposure to poverty impacted maternal mental health. Poverty was described as lack of food in the household, inability to access basic necessities, and limited employment opportunities for women. One CHW described poverty in their community and country:

"Jobs pay very low wages ... I have known mothers with
babies less than one year old whose only chance to earn
money is the coffee harvest. They work while their child
is in a hammock between trees. They stop to breastfeed
and continue picking."

\section{STRATEGIES FOR REDUCING POSTPARTUM DISTRESS}

For the question about mitigating postpartum distress and the role of CHWs, themes were: social support for mothers, involvement of intimate partners, and the well-being of CHWs.

Both CHWs and mothers reported CHWs may offer social support to mothers and encourage more involvement from intimate partners. CHWs were also asked what they need to support mothers after childbirth. They responded that they need to manage their own health and well-being. CHWs and mothers provided some different suggestions for improving maternal mental health. Mothers more frequently described the importance of creating economic opportunities for women, improving the quality of and access to health care, and strengthening food security.

Theme 4: Social support for mothers. CHWs and mothers emphasized the need for social support after childbirth. They felt there should be a trustworthy person in a mother's life, such as her mother, partner, friend, or a CHW, who can offer advice if she is struggling. When asked how CHWs may support mothers, participants suggested CHWs visit women, listen to them, and offer advice or motivation when needed. One mother said speaking with someone outside 
the family, like a CHW, would be useful. She gave an example from her own life, after she had a miscarriage:

$$
\begin{aligned}
& \text { "Yes, [speaking to a CHW would be] very important, be- } \\
& \text { cause days and days passed in which I didn't have any- } \\
& \text { one to talk to about what I was feeling. It's true my par- } \\
& \text { ents were there, but it was difficult for me to talk to } \\
& \text { them." }
\end{aligned}
$$

Although there was a general consensus across both groups that CHWs may visit mothers and offer advice, there was less agreement about what types of advice would be the most appropriate. Some suggested that advice consist of listening and providing encouragement. Others felt CHWs should give specific types of advice consistent with religious or cultural values. Due to gossip, both groups believed confidentiality should be prioritized.

Theme 5: Involvement of intimate partners. CHWs and mothers said the active involvement of partners would help reduce postpartum distress. They indicated sadness after childbirth is a concern that affects the entire family. One of the mothers recommended maternal health education should be offered to couples jointly:

"It would be good to give advice to the couple ... As you know, you cannot just talk to the woman, you have to talk to the man so he understands and feels what the woman is feeling [during the time after childbirth]."

Theme 6: Well-being of CHWs. CHWs said their own mental health played a critical role in their ability to support the mental health of mothers. According to one CHW:

\footnotetext{
"It starts with us, with us being strong ... When I feel I can do things, that I can respect the rights of others, that I have value, then I am able to help others. But if I do not feel good psychologically, emotionally, then I will not be able to help other women."
}

Observations made during the focus group interviews revealed the potential benefits of support groups and other resources for CHWs. At the end of each focus group, nearly all CHWs talked about how valuable the conversation was for their personal development and mental health. Participants of one focus group interview said learning about their colleagues' past hardships normalized their own difficult experiences. They reported appreciating having a safe space where they were able to learn about a new topic and all contribute to the discussion. One of the CHWs said the work they do sometimes puts a strain on their familial and marital relationships. She thought the availability of couples counseling for CHWs would be a useful solution to help husbands appreciate the importance of CHWs as community leaders.

\section{DISCUSSION}

This study revealed that postpartum distress was a common experience among mothers living in a rural community in Nicaragua. The CHWs and mothers thought postpartum distress was influenced by limited people to confide in, intimate partner violence, and household poverty. Also, participants felt that CHWs might play an important role in providing social support to mothers and engaging intimate partners to offer assistance. However, results indicated that CHW interventions for postpartum distress must also consider the well-being of CHWs.

The findings inform recommendations regarding the development of $\mathrm{CHW}$ interventions to support Nicaraguan mothers who have recently given birth, especially those who live in small, rural communities. The first recommendation is that CHWs could visit women during the perinatal period to offer advice and support. This is challenging, however, given the relatively low number of CHWs in rural Nicaragua and their competing responsibilities. The CHWs in the current study reported volunteering their time on MINSA vaccination campaigns, encouraging mothers to complete prenatal checks, assisting with the work of local NGOs, and managing their own lives. It might be worthwhile, therefore, to develop an approach for identifying mothers most vulnerable to experiencing postpartum distress. One possibility is for local health clinics and CHWs to work together to identify women who need additional postpartum support. Another strategy is to train CHWs to screen for postpartum distress. This approach has been used in other LMICs, ${ }^{30}$ but such projects often use adapted Western diagnostic tools that may not be culturally relevant.

Another recommendation is to develop guidelines for what types of advice may be most useful to offer rural Nicaraguan mothers. A group of CHWs, mothers, and health professionals may collaborate on such a project. They may identify information and questions that CHWs can use to establish trust and initiate meaningful conversations. Based on the current study, potential questions are: Are you having difficulties with the baby or worries about the baby? What kinds of help are you getting in the house from your partner and/or family? How are you feeling physically and emotionally? It would also be beneficial if guidelines included a list of trainings and resources for CHWs. An example is the Spanish-language manual entitled "Thinking Healthy" which was developed by the Pan-American Health Organization and WHO and may be adapted to train Nicaraguan CHWs in talking with mothers about postpartum mental health. ${ }^{31}$

A third recommendation is to increase involvement of intimate partners and improve the quality of relationships among couples. Participants expressed interest in strategies that improve gender relations, in particular. By learning more about women's experiences, men may gain tools to better support them, especially during pregnancy and early stages of parenthood. CHWs could support educational workshops by recruiting couples, and facilitating the sessions.

These recommendations align with MINSA's emphasis on maternal and child health. Further, MINSA is best positioned to consider how to incorporate recommendations into their regular healthcare trainings and programming given their level of influence over CHW priorities and activities across the country. However, CHW-related interventions for postpartum distress should also be adapted based on local contexts and CHWs' capacity to expand their workloads. As CHWs are volunteers in Nicaragua, it is vital that they be involved in decision-making processes about the scope of their work. 
The current study highlights the needs of CHWs, including resources and strategies for their self-care. Observations from focus group interviews revealed the potential benefits of support groups for CHWs that address the difficulties they face in their professional and personal lives. In some cases CHWs may need additional resources and supports like counseling if they are experiencing significant distress or difficulties in their own marriages. As local NGOs and MINSA look to expanding the role of CHWs, they must prioritize the mental health of CHWs. This may be achieved by setting aside space for promoting empathy, emphasizing the importance of confidentiality, and collectively finding solutions to common difficulties that arise in CHWs' work. It is equally critical that MINSA considers offering compensation to CHWs as the scope of their work expands. Most CHWs in Nicaragua are volunteers. NGOs and other organizations should also develop systems of payment that accurately reflect their contributions to promoting health in rural communities.

Finally, postpartum distress is a complex and multifaceted problem that requires structural changes in Nicaraguan society. Participants reported maternal distress is influenced by social factors, like intimate partner violence and poverty. As in many countries, there is a body of literature on the impacts of a patriarchal society on women in Nicaragua. ${ }^{20,21}$ A large study conducted with 142 rural and urban municipalities documented roughly $37 \%$ of pregnancies were either unwanted or mistimed. Unintended pregnancies were significantly associated with controlling behavior by a partner, sexual abuse, and exposure to physical/ sexual intimate partner violence. ${ }^{22}$ Although Law 779 passed in 2012, the Comprehensive Law Against Violence Against Women, enforcement has been weak in many parts of the country. Moreover, research has indicated some women think the law makes men more violent because they know they will go to jail whether they hit or kill a woman. ${ }^{32}$ Research also points to poverty affecting maternal mental health in Nicaragua. A study in León found mothers in homes that were even mildly food insecure had a $42 \%$ higher rate of mental distress compared to those in food secure households. ${ }^{19}$

\section{LIMITATIONS}

This study had some limitations. First, the study did not include follow-up interviews with participating mothers. Follow-up interviews with mothers may have led to a greater level of confianza (rapport and trust), which may have resulted in conversations that garnered more information. Another limitation was the small sample size of mothers in the community. However, the number of completed interviews provided some saturation of major themes that were also consistent with themes that emerged from focus group interviews conducted with CHWs. The current study also had limited generalizability because of the unique situation of the CHWs in the participating community. While CHWs tend to work similarly with MINSA throughout Nicaragua,
CHWs in the study community had some unique resources and responsibilities that are not necessarily the same for other CHWs. Differences were due to their coordination with a local NGO. Additionally, the current study was restricted to mothers over the age of 18. Future research should include younger mothers given the high prevalence of teenage pregnancy in rural Nicaragua. Research should also be completed with CHWs from more regions of the country.

\section{CONCLUSIONS}

CHWs in rural Nicaragua may play an important role in reducing postpartum distress and supporting women after childbirth. The Nicaraguan Ministry of Health is well positioned to promote these types of interventions given their influence over CHW activities. However, CHWs need resources and supports to conduct their work successfully. In addition, their efforts should exist in tandem with societal changes that impact maternal health including the amelioration of poverty and prevention of violence against women.

\section{ACKNOWLEDGEMENTS}

The authors are grateful to the mothers and CHWs who participated in this study. They also thank Comunidad Connect, an NGO that collaborated with and hosted the lead researcher in the community.

\section{FUNDING}

The Tinker Foundation and the University's Graduate and Professional Student Council funded this research. The content is solely the responsibility of the authors and does not necessarily represent the official views of the funding agencies or the University.

\section{COMPETING INTERESTS}

The authors have completed the Unified Competing Interest form at http://www.icmje.org/coi disclosure.pdf (available on request from the corresponding author) and declare no conflicts of interest.

\section{CORRESPONDENCE TO:}

Anna Louise Steeves-Reece

OHSU-PSU School of Public Health

3181 SW Sam Jackson Park Road

MC: GH230

Portland, OR 97239 USA

steevesr@ohsu.edu 


\section{REFERENCES}

1. Atif N, Lovell K, Rahman A. Maternal mental health: the missing " $\mathrm{m}$ " in the global maternal and child health agenda. Semin Perinatol.

2015;39(5):345-352. doi:10.1053/i.semperi.2015.06.00 $\underline{7}$

2. Miller RL, Pallant JF, Negri LM. Anxiety and stress in the postpartum: is there more to postnatal distress than depression? BMC Psychiatry. 2006;6(1):12. doi:1 $\underline{0.1186 / 1471-244 \mathrm{x}-6-12}$

3. Gress-Smith JL, Luecken LJ, Lemery-Chalfant K, Howe R. Postpartum depression prevalence and impact on infant health, weight, and sleep in lowincome and ethnic minority women and infants. Matern Child Health J. 2012;16(4):887-893. doi:10.100 7/s10995-011-0812-y

4. Stein A, Pearson RM, Goodman SH, et al. Effects of perinatal mental disorders on the fetus and child. Lancet. 2014;384(9956):1800-1819. doi:10.1016/s014 0-6736(14)61277-0

5. Leis JA, Heron J, Stuart EA, Mendelson T. Associations between maternal mental health and child emotional and behavioral problems: does prenatal mental health matter? J Abnorm Child Psychol. 2014;42(1):161-171. doi:10.1007/s10802-01 3-9766-4

6. Myers S, Johns SE. Postnatal depression is associated with detrimental life-long and multigenerational impacts on relationship quality. PeerJ. 2018;6:e4305. doi:10.7717/peeri.4305

7. Fisher J, Cabral de Mello M, Patel V, et al. Prevalence and determinants of common perinatal mental disorders in women in low- and lower-middleincome countries: a systematic review. Bull World Health Organ. 2012;90(2):139-149H. doi:10.2471/blt.1 1.091850

8. Gavin NI, Gaynes BN, Lohr KN, Meltzer-Brody S, Gartlehner G, Swinson T. Perinatal depression: a systematic review of prevalence and incidence. Obstet Gynecol. 2005;106(5, Part 1):1071-1083. doi:10.1097/0 1.aog.0000183597.31630.db

9. Kohn R, Saxena S, Levav I, Saraceno B. The treatment gap in mental health care. Bull World Health Organ. 2004;82:858-866.
10. Barnett ML, Gonzalez A, Miranda J, Chavira DA, Lau AS. Mobilizing community health workers to address mental health disparities for underserved populations: a systematic review. Adm Policy Ment Health. 2018;45(2):195-211. doi:10.1007/s10488-01 7-0815-0

11. American Public Health Association. Community health workers. Published 2018. Accessed February 20, 2018. https://www.apha.org/apha-communities/m ember-sections/community-health-workers

12. Cooper PJ, Tomlinson M, Swartz L, et al. Improving quality of mother-infant relationship and infant attachment in socioeconomically deprived community in South Africa: randomised controlled trial. BMJ. 2009;338(apr14 2):b974. doi:10.1136/bmj.b $\underline{974}$

13. Baker-Henningham H, Powell C, Walker S, Grantham-McGregor S. The effect of early stimulation on maternal depression: a cluster randomised controlled trial. Arch Dis Child. 2005;90(12):1230-1234. doi:10.1136/adc.2005.073015

14. Chibanda D, Shetty AK, Tshimanga M, Woelk G, Stranix-Chibanda L, Rusakaniko S. Group problemsolving therapy for postnatal depression among HIVpositive and HIV-negative mothers in Zimbabwe. J Int Assoc Provid AIDS Care. 2013;13(4):335-341. doi:10.1 $177 / 2325957413495564$

15. Rahman A, Malik A, Sikander S, Roberts C, Creed F. Cognitive behaviour therapy-based intervention by community health workers for mothers with depression and their infants in rural Pakistan: a cluster-randomised controlled trial. Lancet. 2008;372(9642):902-909. doi:10.1016/s0140-6736(0 8)61400-2

16. Perry HB, Zulliger R, Rogers MM. Community health workers in low-, middle-, and high-income countries: an overview of their history, recent evolution, and current effectiveness. Annu Rev Public Health. 2014;35(1):399-421. doi:10.1146/annurev-pub lhealth-032013-182354

17. Verbeek T, Arjadi R, Vendrik JJ, Burger H, Berger MY. Anxiety and depression during pregnancy in Central America: a cross-sectional study among pregnant women in the developing country Nicaragua. BMC Psychiatry. 2015;15(1):292. doi:10.11 86/s12888-015-0671-y

18. Rudkoski AK. Social support and maternal mental health in rural Nicaragua. Published online 2017. 
19. Piperata BA, Schmeer KK, Rodrigues AH, Salazar Torres VM. Food insecurity and maternal mental health in León, Nicaragua: potential limitations on the moderating role of social support. Soc Sci Med. 2016;171:9-17. doi:10.1016/j.socscimed.2016.10.029

20. Lancaster RN. Life Is Hard: Machismo, Danger, and the Intimacy of Power in Nicaragua. University of California Press; 1992.

21. Ellsberg M, Peña R, Herrera A, Liljestrand J, Winkvist A. Candies in hell: women's experiences of violence in Nicaragua. Soc Sci Med. 2000;51(11):1595-1610. doi:10.1016/s0277-9536(00)0 0056-3

22. Salazar M, San Sebastian M. Violence against women and unintended pregnancies in Nicaragua: a population-based multilevel study. BMC Women's Health. 2014;14(1):26. doi:10.1186/1472-6874-14-26

23. Sapag JC, Herrera A, Trainor R, Caldera T, Khenti A. Global mental health: transformative capacity building in Nicaragua. Glob Health Action. 2013;6(1):21328. doi:10.3402/gha.v6i0.21328

24. Nicaragua WHO. Published online 2018. http://ww w.who.int/countries/nic/en/

25. Rodríguez JJ, Barrett T, Narváez S, Caldas JM, Levav I, Saxena S. Sistemas de salud mental en El Salvador, Guatemala y Nicaragua: Resultados de una evaluación mediante el WHO-AIMS. Rev Panam Salud Publica. 2007;22(5):348-357. doi:10.1590/s1020-4989 2007001000008
26. Garfield RM, Taboada E. Health services reforms in revolutionary Nicaragua. Am J Public Health. 1984;74(10):1138-1144. doi:10.2105/ajph.74.10.1138

27. Adamo M, Rivera D, Shah R, Koepsell J, Martínez L, Ortiz JP. Time volunteered on community health activities by brigadistas in Nicaragua. Rev Panam Salud Publica. 2016;40:388-395.

28. Kvernflaten B. Meeting targets or saving lives: maternal health policy and Millennium Development Goal 5 in Nicaragua. Reprod Health Matters. 2013;21(42):32-40. doi:10.1016/s0968-8080(13)4272 $\underline{8-3}$

29. de Reconciliación G, Nacional U. Instituto Nacional de Información de Desarrollo INIDE. Anuario Estadístico; 2015. Accessed February 15, 2018. htt p://www.inide.gob.ni/Anuarios/Anuario\%20Estadistic o\%202015.pdf

30. Hung KJ, Tomlinson M, le Roux IM, Dewing S, Chopra M, Tsai AC. Community-based prenatal screening for postpartum depression in a South African township. Int J Gynaecol Obstet. 2014;126(1):74-77. doi:10.1016/j.ijgo.2014.01.011

31. World Health Organization, Pan American Health Organization. Pensamiento saludable: manual para la atención psicosocial de la depresión perinatal. Published 2015. Accessed February 10, 2018. http://iri s.paho.org/xmlui/handle/123456789/28417

32. Luffy SM, Evans DP, Rochat RW. “Siempre me critican": barriers to reproductive health in Ocotal, Nicaragua. Rev Panam Salud Publica. 2015;37(4-5):245-250. 